\title{
Bibliometric Analysis of Scientific Productivity around Edge Computing and the Internet of Things
}

\author{
Antonio-José Moreno-Guerrero $₫$, Francisco-Javier Hinojo-Lucena $₫$, \\ Magdalena Ramos Navas-Parejo and Gerardo Gómez-García * \\ Department of Didactics and School Organization, University of Granada, 51001 Ceuta, Spain; \\ ajmoreno@ugr.es (A.-J.M.-G.); fhinojo@ugr.es (F.-J.H.-L.); magdalena@ugr.es (M.R.N.-P.) \\ * Correspondence: gomezgarcia@ugr.es
}

Received: 14 September 2020; Accepted: 16 November 2020; Published: 17 November 2020

\begin{abstract}
Technological progress has recently led to the emergence of various technological resources and means that are improving specific aspects of society. An example of this can be found in the "internet of things" and "edge computing". The present study aims at knowing and analyzing the scientific literature of the set of terms formed by "edge computing" and "internet of things", called from now on ECIT. In order to carry out the research, a study has been developed, based on bibliometrics, by means of scientific mapping. In this case, different production indicators have been taken into account, as well as the structural and dynamic development of the terms and authors extracted from the publications through the programs Analyze Results, Creation Citation Report and SciMAT. The results indicate that the study theme "edge computing" and "internet of things" is of recent creation, given that its beginnings date back to 2014. Since then the level of production has been dizzying, increasing considerably in the past two years. It can be concluded that the field of study of ECIT is of recent creation, with a solid research base based on the "internet of things". Furthermore, the themes "big data", "energy" and "framework" can be considered as the future lines of research on ECIT.
\end{abstract}

Keywords: edge computing; internet of things; bibliometric; SciMAT

\section{Introduction}

In recent years we have seen a progressive development of information technology, as well as an enormous number of intelligent devices and objects that are monopolizing our daily lives [1]. With the recent developments in sensor, communication and microcontroller technologies, we are witnessing a convergence of the physical world and the cyber world $[2,3]$.

This phenomenon has culminated in the integral concept of the internet of things (IoT), which is present in many of the devices we find in our environment: intelligent cars, portable computers, sensors, industrial components, etc. [4-6], and which aims to build an intelligent world that allows us to perceive, analyze, control and optimize traditional physical systems using cybernetic technologies [7]. The International Telecommunication Union (ITU) defines IoT as "a global infrastructure for the information society, enabling advanced services through the interconnection (physical and virtual) of things based on existing and evolving interoperable information and communication technologies" [8]. In this sense, [9] coined the term "internet of things" to describe the network that connects objects in the physical world to the internet [10-12].

However, among the limitations of the IoT is the low storage and processing capacity that limits the performance of the service [13]. Given this situation, the emergence of edge computing has been presented as a possible solution to provide better device performance in terms of latency, low operational cost, high stability and better quality of service [14]. 
Edge computing is proposed in order to move computing capacity from centralized cloud servers to edge nodes near the user end. The inclusion of this phenomenon entails some improvements with respect to existing devices [15]:

- Firstly, the edge nodes can pre-process large amounts of data before transferring it to the central servers in the cloud, and

- Cloud resources are optimized by enabling compute-capable edge nodes.

Compared to cloud computing, it provides shorter response times and greater reliability [16]. Furthermore, to save bandwidth and reduce latency, more data is processed at the edge instead of uploading it to the cloud [17].

Therefore, edge computing allows data produced by IoT devices to be processed closer to where they were created, rather than sending them over long distances to reach data centers and computing clouds [18]. As a consequence, this results in the devices being able to analyze the relevant data with minimal latency, which is a necessity in many companies and institutions of different nature $[19,20]$. Such is the impact of edge computing on society that the McKinsey Global Institute estimates that the total economic impact of IoT and edge computing devices will reach 11 billion dollars in 2025 [21].

Edge computing follows a decentralized architecture with data processing at the edge of the border network nodes to make autonomous decisions. Therefore, applications running on edge computing will perform actions locally before connecting to the cloud, thus reducing network overload problems, as well as possible drawbacks in terms of security and privacy. Likewise, this network can be easily integrated with other wireless networks such as ad hoc mobile networks (MANETs), vehicle networks (VANETs), intelligent transport systems (ITSs) or IoT to mitigate network and computer-related problems $[22,23]$. The link with edge computing provides network applications with an improvement in their decision-making speed [14].

In this sense, the scientific literature shows several studies in which the effectiveness of the application of edge computing in different IoT devices is determined, ranging from vehicle traffic control and congestion [16,24] to intelligent vehicles [25-27], and in tele-medical devices, in which their effectiveness in detecting possible disorders or illnesses in people, especially the elderly, is observed [28-31], among other applications.

Therefore, it is a modern line of research, which attracts the interest of numerous experts from different branches of knowledge. Based on these precedents, the present study presents knowledge and analysis of the scientific literature of the set of terms formed by "edge computing" and "internet of things", called from now on ECIT. To carry out the research, a study has been developed, based on bibliometrics, by means of scientific mapping. In this case, different production indicators have been taken into account, as well as the structural and dynamic development of the terms and authors extracted from the publications.

The research procedure developed followed the structure and steps carried out in other bibliometric-based research, and was carried out in the Web of Science (WoS) database, as it is a model that has already been validated [32-35].

With all this, the purpose of this research is to know the state of the art of the term ECIT in the WoS database. The following specific objectives are based on this general objective:

- To know the performance of the scientific production of ECIT.

- Determine the scientific evolution of ECIT.

- To identify the most influential themes in ECIT publications.

- Find out about ECIT's top authors. 


\section{Materials and Methods}

\subsection{Research Design}

The research design has been based on scientometrics, specifically bibliometric studies. In this study, processes of search, registration, analysis and prediction of scientific terms have been carried out [36]. To do so, the guidelines set by experts in this type of study have been followed [37].

More specifically, the research was carried out based on an analysis of co-words [38], taking into account various bibliometric indicators (h, $\mathrm{g}, \mathrm{hg}, \mathrm{q} 2$ index) $[39,40]$. Thanks to this, it has been possible to generate thematic maps on the yield and location of the conceptual subdomains [41] related to ECIT. In addition, it has been possible to establish the thematic development of the term ECIT in the scientific literature [42].

\subsection{Procedure and Data Analysis}

This study has followed several phases. First, the database was determined. In this case, WoS was chosen because it is a database where relevant literature is stored, and it is also the database from which Journal Citation Reports (JCR) feeds in order to establish the most relevant metrics in the field of research. Next, the search of all the scientific literature on ECIT metadata was carried out, using the following search equation: "edge computing" [TITLE] AND "internet of things" [TITTLE]. This search initially generated a total of 1331 documents of various kinds. After performing and applying a PRISMA protocol (Figure 1), a total of 1171 remained. These documents are the ones with which the present study was finally carried out. As criteria for inclusion, all the existing scientific production in WoS has been taken into account, except for the scientific production of the year 2020. As an exclusion criterion, in addition to the year 2020, we added the literature that appears in the WoS.

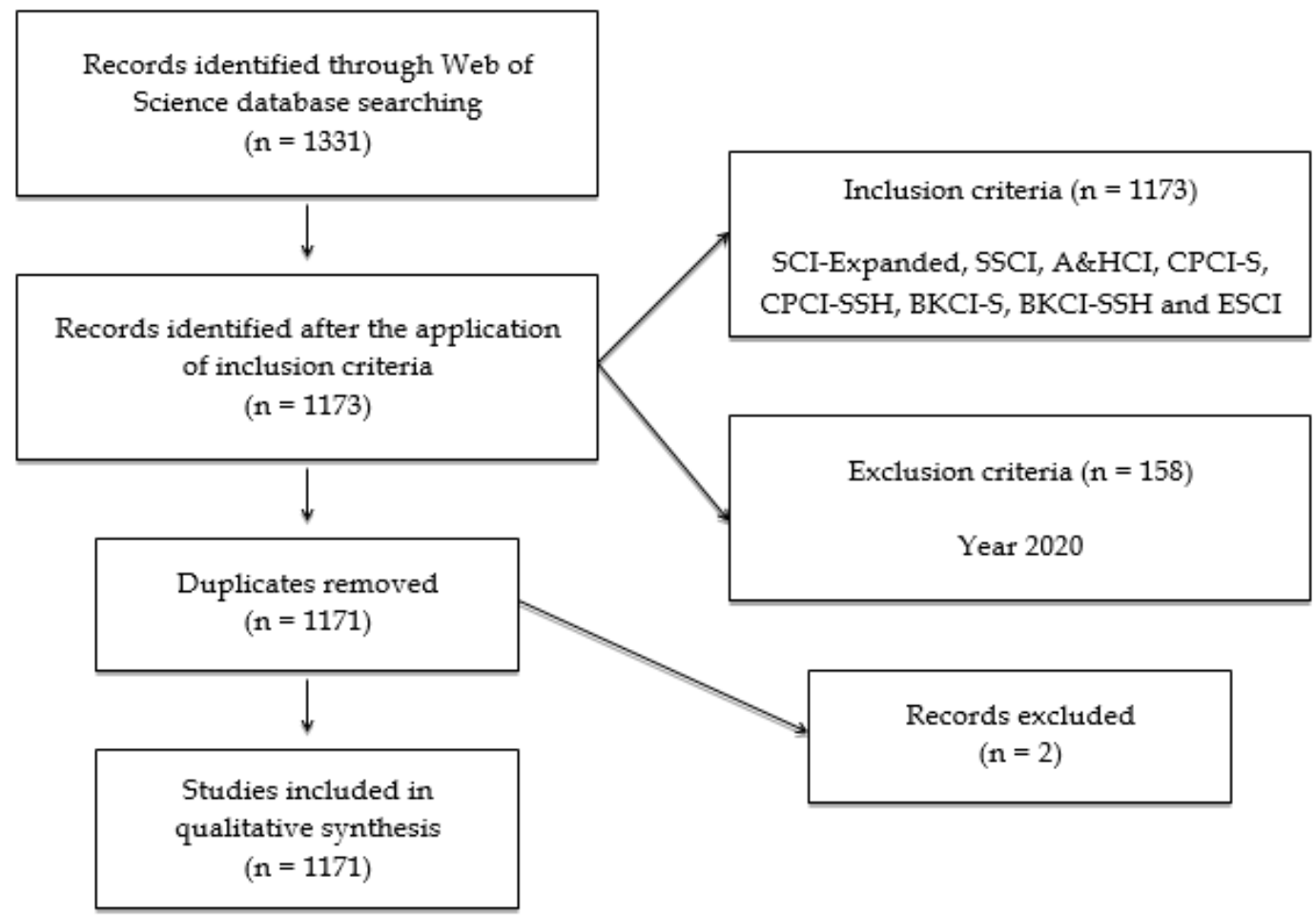

Figure 1. Flow chart according to the PRISMA protocol.

The analysis of the term ECIT has been carried out through various programs. On the one hand, for the descriptive analysis, the programs Analyze Results and Creation Citation Report have been used. These tools are already incorporated into WoS. This has served to identify the following variables, with the corresponding indicators of inclusion: (a) year of publication (2014-2019); (b) language (all 
production); (c) area of knowledge ( $x \geq 100)$; (d) type of document ( $x \geq 30)$; (e) authors $(x \geq 10)$; (f) journals ( $x \geq 25)$; (g) institution ( $x \geq 20)$; (h) country ( $x \geq 70)$; (i) 4 most cited documents ( $x \geq 420)$.

For the analysis of academic performance, and of authors, the SciMAT software has been used, identifying, in a longitudinal way, the structural and dynamic development of the scientific production. With SciMAT, a co-word analysis has been carried out at a thematic level, and of authors, bearing in mind the following phases $[43,44]$ :

Recognition: In this phase the key words of the reported documents are analyzed $(n=1366)$. A map of co-occurrence nodes is generated. A standardized network of co-words is elaborated. The most significant keywords $(n=1332)$ are detected, after a debugging procedure, in which the keywords appearing in singular and plural are grouped together. In this study no modifications of the key words have been established, in order to avoid the bias of the researchers themselves. The most determining topics and concepts are represented by a clustering algorithm.

Reproduction: A strategic diagram and a thematic network are drawn up according to the principles of centrality and density. The resulting diagram distributes the most relevant themes as follows: 1-Upper right for motor and relevant themes; 2-Upper left for rooted and isolated themes; 3-Lower left for disappearing or projected themes; 4-Lower right for underdeveloped and cross-cutting themes.

Determination: The evolution of the themes in the different established periods of time is identified. In this research three periods have been configured $(\mathrm{P} 1=2014-2017 ; \mathrm{P} 2=2018 ; \mathrm{P} 3=2019)$. For the authorship all the literary production in WoS has been taken into account, conforming to a single period ( $P X=2014-2019$ ). The strength of association is calculated through the volume of keywords or themes in common between the different periods.

Performance: In this phase, in which the different results are obtained, various production indicators have been taken into account (Table 1).

Table 1. Output indicators and inclusion criteria for the performance phase.

\begin{tabular}{cc}
\hline Configuration & Values \\
\hline Analysis unit & Keywords authors, keywords WoS \\
Frequency threshold & Keywords: $\mathrm{P}_{1}=(2), \mathrm{P}_{2}=(2), \mathrm{P}_{3}=(2)$ \\
Network type & Authors: $\mathrm{P}_{X}=(3)$ \\
& Co-occurrence \\
Co-occurrence union value threshold & Keywords: $\mathrm{P}_{1}=(2), \mathrm{P}_{2}=(2), \mathrm{P}_{3}=(2)$ \\
Normalization measure & Authors: $\mathrm{P}_{X}=(3)$ \\
Clustering algorithm & Equivalence index \\
Evolutionary measure & Maximum size: 9 ; Minimum size: 3 \\
Overlapping measure & Jaccard index \\
& Inclusion Rate \\
\hline
\end{tabular}

\section{Results}

\subsection{Scientific Performance and Production}

The scientific evolution of the 1171 documents on the ECIT theme shows a dizzying evolution in recent years. It is of recent creation, given that its beginnings in WoS date back to 2014. From that date until 2016, production maintains a moderate rise, but from 2017 to 2019, production shoots up. This shows a great impact on the scientific community of ECIT studies (Figure 2).

The language used by the scientific community to present scientific findings is English ( $n=1170)$. Only one production is known to be in Turkish $(n=1)$.

The area of knowledge where the studies on ECIT are collected is divided mainly between electrical electronic engineering and telecommunications, which marks its clear scientific profile in the field of electronics and communication (Table 2). 


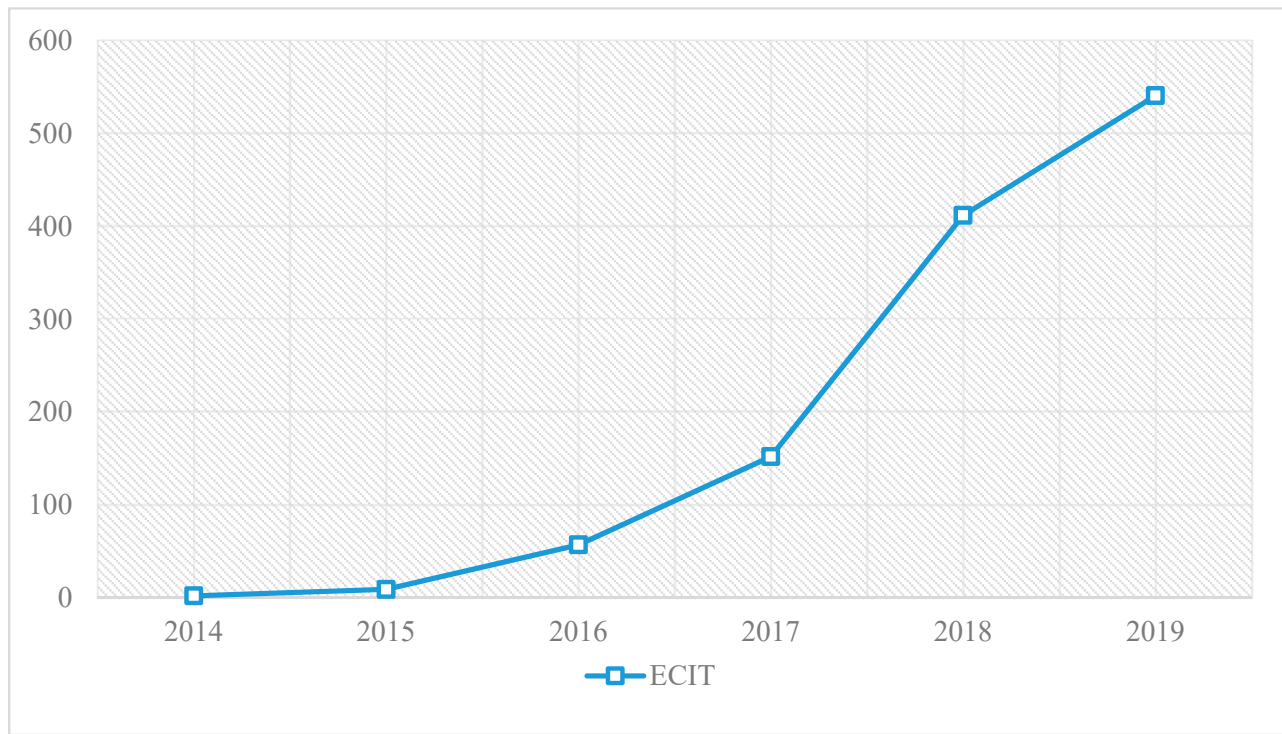

Figure 2. Evolution of scientific production.

Table 2. Areas of knowledge.

\begin{tabular}{cc}
\hline Area of Knowledge & ECIT \\
\hline Engineering electrical electronic & 657 \\
Telecommunications & 514 \\
Computer science information systems & 438 \\
Computer science theory methods & 333 \\
Computer science hardware architecture & 160 \\
\hline
\end{tabular}

The type of document most used by the authors to present the results achieved in their research is articles, although it is closely followed by proceedings papers. This is due to the fact that this is a topic that has recently appeared in the scientific community, given that the first findings are usually shown in conference communications (Table 3 ).

Table 3. Type of document.

\begin{tabular}{cc}
\hline Document Type & ECIT \\
\hline Article & 617 \\
Proceedings paper & 518 \\
Review & 33 \\
\hline
\end{tabular}

The author with the most scientific production on ECIT is Zhang, Y., although he is closely followed by Chen, $Y$. In this section no pressing production differences are shown, since there are many authors with even production levels (Table 4).

Table 4. Authors.

\begin{tabular}{cc}
\hline Authors & ECIT \\
\hline Zhang Y. & 13 \\
Chen Y. & 11 \\
Dustdar S. & 10 \\
Guerrieri A. & 10 \\
Guizani, M. & 10 \\
Lui, J.J. & 10 \\
Wang X. & 10 \\
\hline
\end{tabular}


The journal with the most production on the subject is IEEE Access, followed closely by IEEE Internet of Things Journal. In this case, the IEEE consortium is a reference in this field of study (Table 5).

Table 5. Journals.

\begin{tabular}{cc}
\hline Journal & ECIT \\
\hline IEEE Access & 101 \\
IEEE Internet of Things Journal & 97 \\
Sensors & 47 \\
Future Generation Computer System the International Journal of Science & 38 \\
\hline
\end{tabular}

The institution with the most production on the subject is Beijing University of Posts and Telecommunications, followed by the Chinese Academy of Sciences (Table 6).

Table 6. Institutions.

\begin{tabular}{cc}
\hline Institutions & ECIT \\
\hline Beijing University of Posts and Telecommunications & 50 \\
Chinese Academy of Sciences & 32 \\
Xidian University & 26 \\
University of Electronic Science Technology of China & 23 \\
University of Messina & 21 \\
University system of Georgia & 21 \\
\hline
\end{tabular}

The country with the largest amount of production is China, followed by the USA. The rest of the countries show production levels that are distant from these two countries (Table 7).

Table 7. Country.

\begin{tabular}{cc}
\hline Country & ECIT \\
\hline China & 352 \\
USA & 267 \\
Italy & 97 \\
Canada & 81 \\
South Korea & 78 \\
India & 73 \\
England & 72 \\
\hline
\end{tabular}

The most cited publication is that of Shi et al. (2016), with a high level of citations, totaling 929. This shows the high interest in this subject on the part of the scientific community, so a high production is expected in the next years (Table 8).

Table 8. Edge computing and internet of things (ECIT): most cited references.

\begin{tabular}{|c|c|}
\hline References & Citations \\
\hline $\begin{array}{c}\text { Shi, W.; Cao, J.; Zhang, Q.; Li, Y.H.Z.; Xu, L.Y. Edge Computing: Vision and Challenges. } \\
\text { IEEE Internet of Things Journal 2016, 3, 637-646. }\end{array}$ & 929 \\
\hline $\begin{array}{c}\text { Chiang, M.; Zhang, T. Fog and IoT: An Overview of Research Opportunities. IEEE Internet } \\
\text { of Things Journal 2016, 3, 854-864. }\end{array}$ & 616 \\
\hline $\begin{array}{c}\text { Mao, Y.Y.; You, C.S.; Zhang, J.; Huang, K.B.; Letaief, K.B. A Survey on Mobile Edge } \\
\text { Computing: The Communication Perspective. IEEE Communications Surveys and Tutorials } \\
\text { 2017, 19, 2322-2358 }\end{array}$ & 532 \\
\hline $\begin{array}{c}\text { Lin, J.; Yu, W.; Zhang, N.; Yang, X.Y.; Zhang, H.L.; Zhao, W. A Survey on Internet of Things: } \\
\text { Architecture, Enabling Technologies, Security and Privacy, and Applications. IEEE Internet } \\
\text { of Things Journal 2017, 4, 1125-1142. }\end{array}$ & 424 \\
\hline
\end{tabular}




\subsection{Structural and Thematic Development}

The evolution of keywords, which is represented in Figure 3, shows information about the keywords that enter and leave in the previously established periods. In addition, it shows the level of coincidence of keywords between periods. In this case, it is shown that between the first and second period, the level of keyword matching is 36\%, while between the second and third period it is $31 \%$. Although the percentages of coincidence are high, there is a downward trend. This is because the ECIT study is establishing new lines of research.

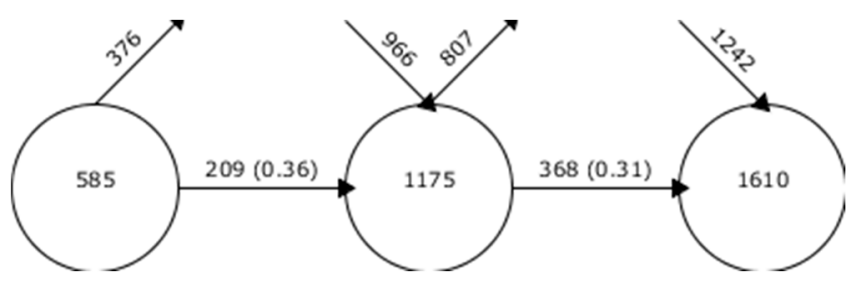

Figure 3. Continuity of keywords between contiguous intervals.

The academic performance of each of the established periods indicates the most relevant topics, taking into account various bibliometric indicators. Bearing in mind what is indicated in Table 9, in the first period (2014-2017), the subject matter with the best bibliometric indicators is "things". In the second period (2018) and in the third period (2019), the theme with the highest bibliometric value is "internet of things". This shows that the current relevance of ECIT is in its own name. It can be interpreted that as it is a recently created term, research still focuses on its delimitation and denomination.

Table 9. Thematic performance in ECIT.

\begin{tabular}{ccccccc}
\hline Denomination & Works & $\begin{array}{c}\text { Period 2014-2017 } \\
\text { Index h }\end{array}$ & Index & Index ho & Index q & Citations \\
\hline Things & 72 & 23 & 45 & 32.17 & 31.81 & 2110 \\
Management & 18 & 12 & 17 & 14.28 & 24 & 1144 \\
Edge-computing & 19 & 6 & 14 & 9.17 & 15.49 & 209 \\
SDN & 8 & 5 & 6 & 5.48 & 15.49 & 216 \\
System & 6 & 5 & 6 & 5.48 & 15.81 & 581 \\
Complex-event-processing & 2 & 1 & 1 & 1 & 1.73 & 3 \\
Wireless-Energy-Transfer & 2 & 0 & 0 & 0 & 0 & 0 \\
Scheme & 3 & 3 & 3 & 3 & 18.81 & 584 \\
Computation-offloading & 3 & 2 & 3 & 2.45 & 7.62 & 33 \\
\hline & & Period 2018 & & & \\
Denomination & Works & Index h & Index g & Index hg & Index q2 & Citations \\
\hline Resource-allocation & 12 & 6 & 8 & 6.93 & 21.63 & 389 \\
Internet-of-things & 213 & 25 & 45 & 33.54 & 37.75 & 2442 \\
Challenges & 16 & 10 & 16 & 12.65 & 17.32 & 449 \\
Architecture & 12 & 6 & 11 & 8.12 & 124.25 & 538 \\
System & 16 & 10 & 16 & 12.65 & 13.04 & 495 \\
5G & 7 & 3 & 6 & 4.24 & 4.9 & 49 \\
Automation-and-control & 2 & 1 & 1 & 1 & 2 & 4 \\
Wavelet & 3 & 1 & 1 & 1 & 3.46 & 12 \\
SDN & 4 & 3 & 3 & 3 & 6.24 & 105 \\
Big-data & 9 & 4 & 7 & 5.29 & 9.17 & 95 \\
Data-box & 2 & 1 & 1 & 1 & 1 & 1 \\
Authentication & 4 & 4 & 4 & 4 & 12.17 & 169 \\
Named-data-networking & 3 & 2 & 3 & 2.45 & 3.16 & 10 \\
Virtualization & 5 & 4 & 5 & 4.47 & 6.93 & 49 \\
Renewable-energy & 3 & 2 & 2 & 2 & 5.83 & 25 \\
Mobile & 3 & 2 & 3 & 2.45 & 11.49 & 78 \\
\hline & & & & & & \\
\hline
\end{tabular}


Table 9. Cont.

\begin{tabular}{ccccccc}
\hline & \multicolumn{6}{c}{ Period 2019} \\
Denomination & Works & Index h & Index g & Index hg & Index q & Citations \\
\hline Internet-of-things & 347 & 16 & 23 & 19.18 & 20 & 1193 \\
Communication & 21 & 7 & 9 & 7.94 & 9.17 & 104 \\
Computation-offloading & 34 & 7 & 11 & 8.77 & 7.48 & 156 \\
Technologies & 10 & 5 & 6 & 5.48 & 6.71 & 45 \\
5G & 15 & 3 & 7 & 4.58 & 6.48 & 56 \\
Edge & 19 & 7 & 11 & 8.77 & 9.54 & 127 \\
Big-data & 9 & 4 & 5 & 4.47 & 8.25 & 64 \\
Mobile-edge-computing & 12 & 6 & 9 & 7.35 & 7.35 & 85 \\
Blockchain & 25 & 8 & 11 & 9.38 & 10.2 & 149 \\
Framework & 10 & 3 & 4 & 3.46 & 3 & 26 \\
Task-analysis & 6 & 1 & 3 & 1.73 & 4.47 & 22 \\
Energy & 6 & 3 & 3 & 3 & 3 & 14 \\
Extreme-learning-machine & 2 & 1 & 1 & 1 & 2.24 & 5 \\
Smart-environments & 3 & 2 & 2 & 2 & 3.16 & 7 \\
Genetic-algorithm & 3 & 2 & 3 & 2.45 & 3.46 & 11 \\
\hline
\end{tabular}

The interval diagrams presented show information about the importance of each of the themes. This is achieved through a clustering mechanism. The Callon indicator is taken into account for this purpose. This indicator analyzes the level of connection of a thematic network with respect to other networks, from two angles: centrality, which measures the strength of external links with other topics, being the measure of the importance of a topic in the development of a certain field of research; and density, which analyzes the internal strength of the network, identifying the internal links between all the key words that are grouped around a specific topic, thus offering the degree of development of the field of study analyzed (Figure 4).

In the first period (2014-2017), the driving themes are "things", which relates to "cloud-computing", "fog-computing", "security", "internet-of-things", "sensors", "service", "smart-city" and "internet"; and "sdn", which relates to "mec", "openflow", "challenges", "nfv" and "5G". In this first period, it is shown how the trend in research is oriented towards the possibilities offered by the internet for storing information, security and access to mobile networks.

In the second period (2018), the driving theme is "internet-of-things", which is related to "fog-computing", "security", "things", "cloud", "edge-computing", "internet", "blockchain" and "cloud-computing". In this case, it follows the trend of the first period, continuing to focus research on the use of the internet for information storage and security.

In the third period (2019), the driving themes are "internet-of-things", which is related to "fog-computing", "security", "things", "cloud", "edge-computing", "resource-allocation", "internet" and "cloud-computing"; "communication", which relates to "wireless-energy-transfer", "maximization", "trajectory-design", "networks", "system", "power-allocation", "computation" and "transmission"; "technologies", which relates to "IIOT", "smart-factory", "simulation". "cyber-physical-system", "protocol", "vanet, "fog" and "industry 4.0"; and "computation-offloading", which relates to "game-theory", "execution", "stochastic-optimization", "mobile-edge-computing", "application-partitioning", "optimization", "management" and "radio". In other words, it follows the same trend as in previous periods, although the scientific community is beginning to open up the field of research to other topics directly related to connection networks and transmission media. Furthermore, in this period, the "big-data", "energy" and "framework" themes must be taken into account. These themes are considered as unknowns, due to their location in the diagram. This indicates to us that they can be the driving themes on the ECIT theme in the coming years. 


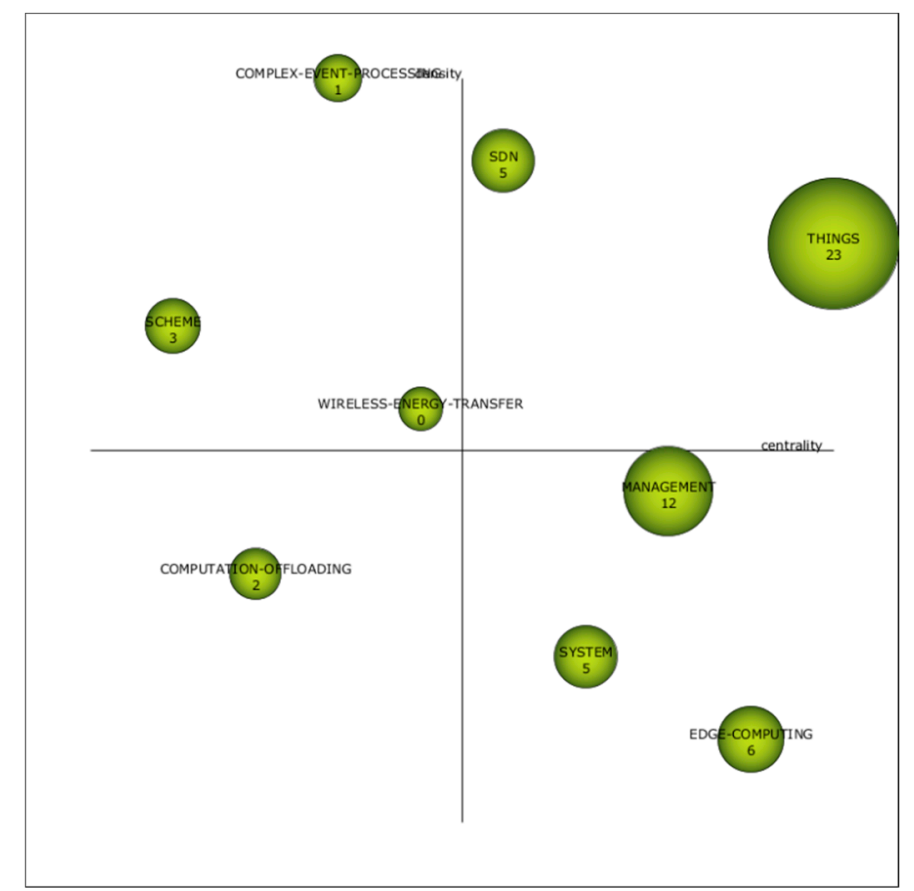

(a)

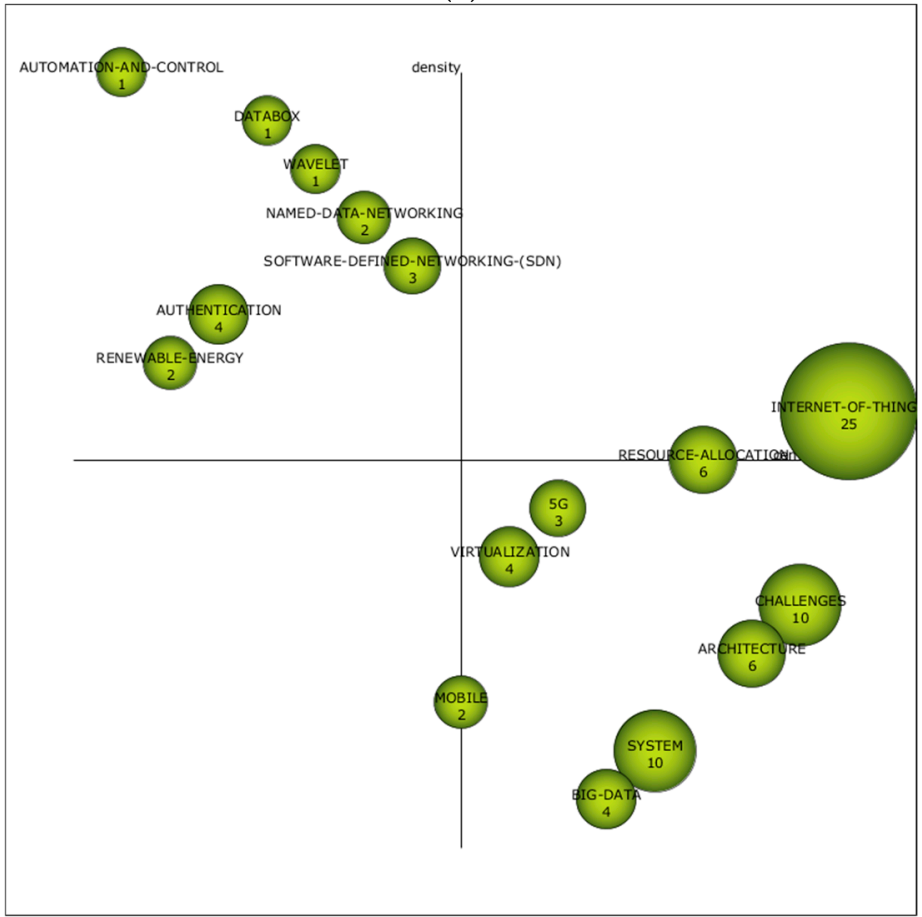

(b)

Figure 4. Cont. 


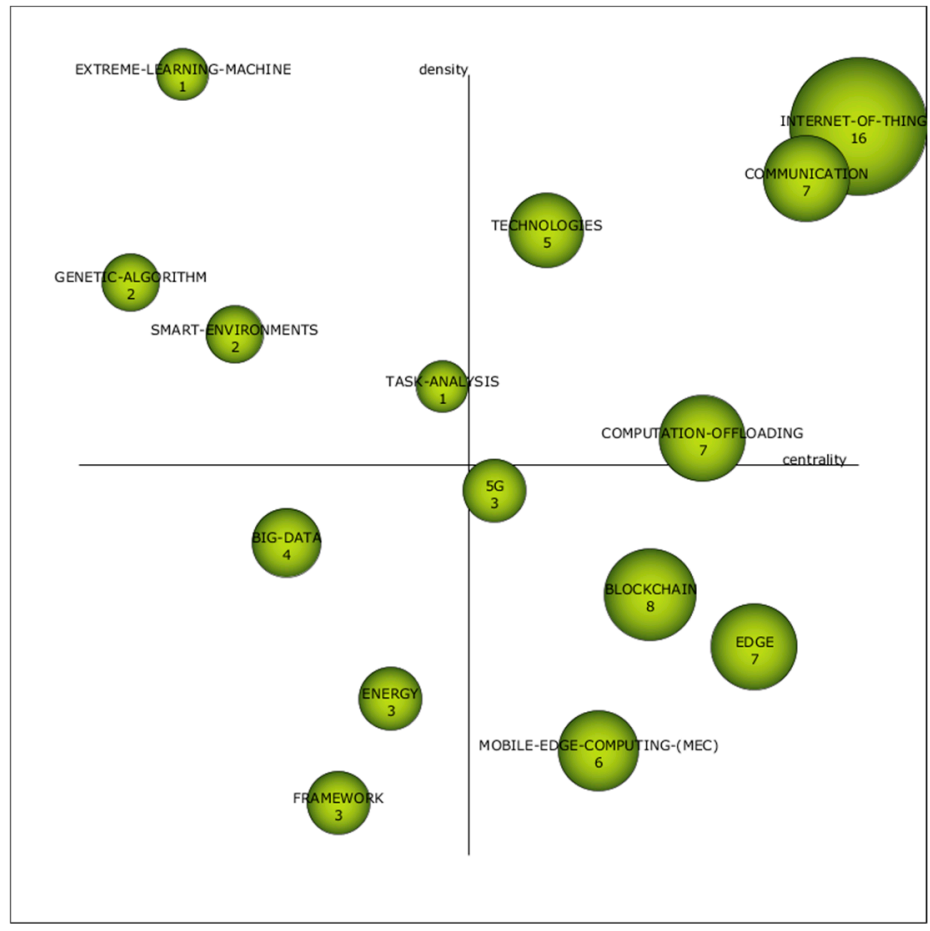

(c)

Figure 4. Strategic diagram by ECIT h-index: (a) interval 2014-2017; (b) interval 2018; (c) interval 2019.

\subsection{Thematic Evolution of Terms}

The thematic development shows the strength of association established between the different themes of the established time periods. To this end, the Jaccard index is taken into account. The development is generated if in a certain time period key words, or themes, are shared with the contiguous time periods. The more key words or themes are related, the stronger and more solid the evolution is. The two types of connections that can occur are: thematic, which is represented by a continuous line; and keyword, which is represented by a discontinuous line.

If we take into account the data reached in Figure 5, we can see that there is a conceptual gap. That is to say, there is not a theme that is repeated in the three periods. This does not mean that there is not a solid line of research. On the contrary, in this case, the ECIT field of study shows a solid line of research, based on time, such as "things_internet-of-things". In this case, the line is more solid between the second and third period than between the first and second period. In addition, between the periods there are more continuous than discontinuous lines. This indicates to us that there is an established research base, whose lines of investigation are connected. 


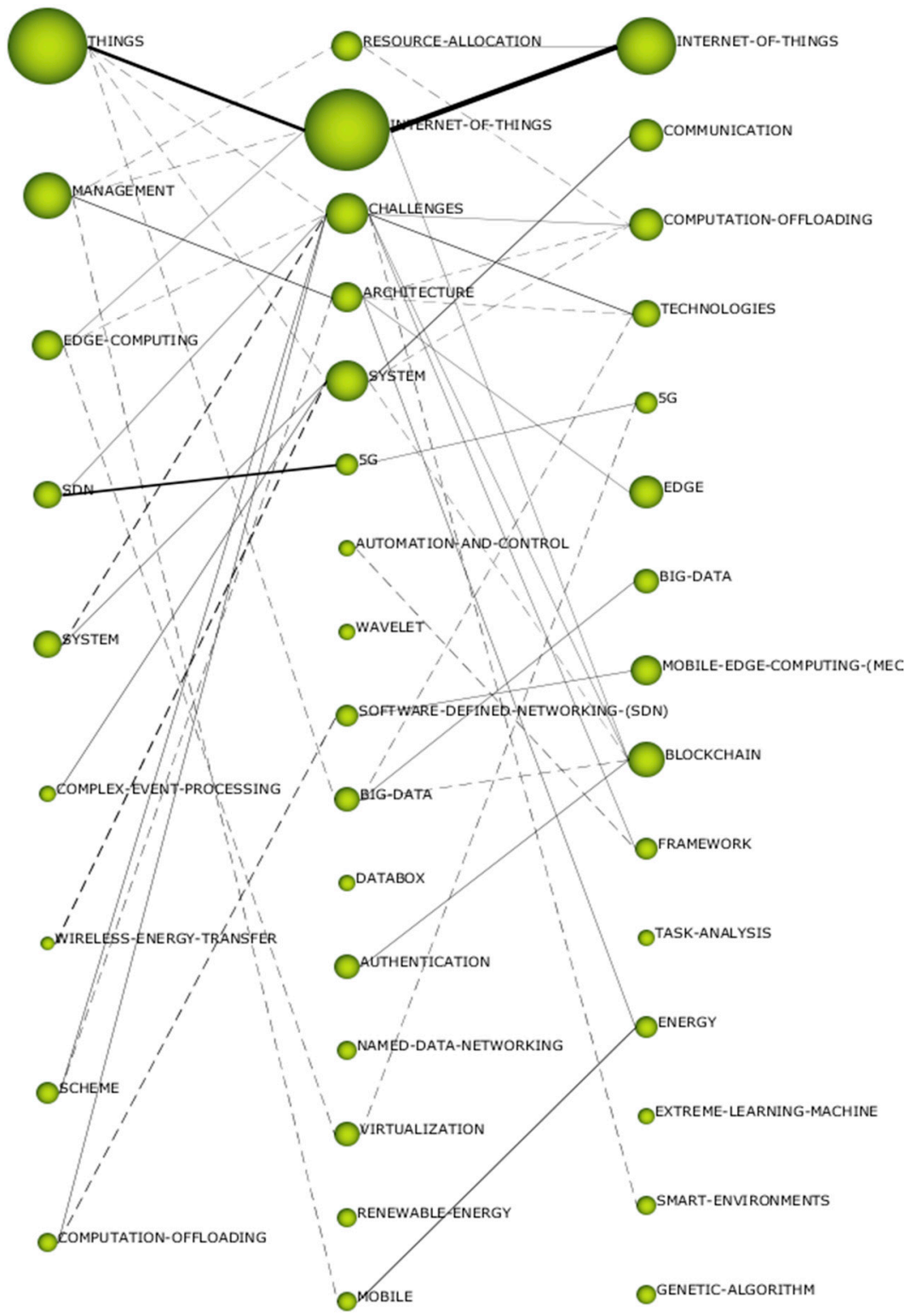

Figure 5. Thematic evolution by h-index.

\subsection{Authors with the Highest Relevance Index}

Taking into account the data collected in Figure 6, it can be seen that the driving authors on the ECIT theme are Cicirelli, F., Nakamura, Y., Xiong, Z.H., and Kong, Y. In addition, the authors Liu, J.J., Alogaily, M., and Cho, S., can be considered the most relevant authors in this field of study. 


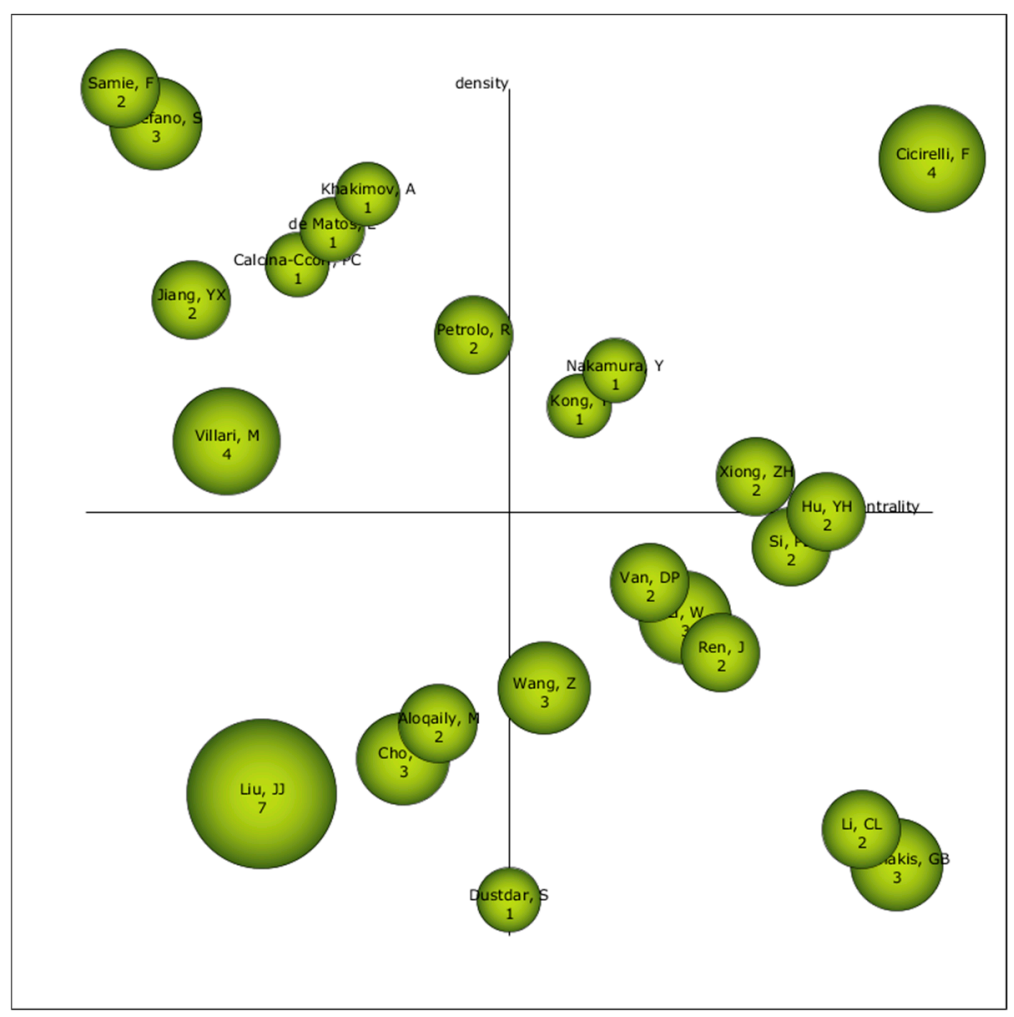

Figure 6. Strategic author diagram of the entire production.

\section{Discussion}

The emergence of edge computing has meant a revolution in the technological landscape. Its inclusion in the various IoT devices has led to an optimization of the performance of these tools in terms of data processing, storage, cost and, above all, latency terms [1,2].

The impact of this phenomenon has also been felt by the scientific community, which, as this study shows, has been commissioned to propose various research projects on this type of technology and on the multiple effects of its application in different types of intelligent devices [3,5].

Based on the results achieved in this research, it can be indicated that the ECIT study theme is of recent creation, given that its beginnings date back to 2014. Since then, the level of production has been dizzying, increasing considerably in the past two years.

In addition, with the data collected, it is possible to make an X-ray of ECIT production, being able to indicate that the documents used to present the scientific results are research articles, written in English, with the main knowledge area being electrical electronic engineering. The magazine where most research is published about ECIT is IEEE Access, the institution is Beijing University of Posts and Telecommunications and the country China, the most prolific in production level. The most cited reference is that of Shi et al. (2016), with the author Zhang, Y. being the most productive, and the authors Cicirelli, F., Nakamura, Y., Xiong, Z.H., and Kong, Y. the most relevant in the subject of study.

With regard to the evolution of the subject, it is observed that it has a solid research base, although in recent times new trends have been observed in the field of research on ECIT. An example of these is the bibliometric values of each of the periods analyzed. In the first period, between 2014 and 2017, the theme with the highest value is "thing", while in the years 2018 and 2019, it is "internet-of-things". If we look more closely at the level of relevance of each of the themes, we can see that in the first and second periods, the trend in research is towards the possibilities offered by the internet for storing information, security and access to mobile networks. On the other hand, in the third period, although it maintains the line of investigation of the two previous periods, new trends are observed, such as connection networks and means of transmission. It should be noted that the themes with the greatest 
bibliometric value are usually the driving forces of each of the periods. This fact is not very commonly acknowledged if research with similar characteristics is observed. This corroborates that there is a solid research base.

The "big-data", "energy" and "framework" themes should also be borne in mind, since they may be the new trends in the field of ECIT research. In addition, it can be indicated that the thematic evolution is based on "internet-of-things", since it is the one that marks the base of evolution of this field of study. This shows that it is a young field of research, which today is settling the scientific limitations of study.

\section{Conclusions}

It can be concluded that the field of study of ECIT is of recent creation, with a solid research base based on "internet-of-things". Furthermore, the "big-data", "energy" and "framework" themes can be considered as the future lines of research on ECIT.

The purpose of this research is to offer researchers the new trends in the near future on the most relevant and interesting topics for the scientific community, as well as to show the aspects on which research has been based in recent times, so that they can have a basis on which to start, develop or guide their studies. It also provides experts in this field with an insight into the current state of research on edge computing and IoT. Finally, the evolutionary analysis has detected those changes and modifications that research on this topic has undergone over the years.

This study presents several research limitations. Firstly, there is the purification of the data presented in WoS, where repeated documents are presented or which are not related to the subject of the study, which requires an effort and great concentration on the part of the researchers to analyze the high number of documents found. Secondly, the establishment of the intervals, which in this case is a question of equity, given that the researchers have sought at all times to maintain a similar number of documents in each of the intervals. Thirdly and finally, the parameters marked in this study have been established according to the researchers' own criteria; they have sought to present results in accordance with their size and relevance. For this reason, the data presented here should be analyzed with caution, given that the change in the parameters established in this research may cause the number and connections in the subjects presented to vary. As future lines of research, we plan to carry out research on ECIT associated with big data.

Author Contributions: Conceptualization, F.-J.H.-L.; methodology, M.R.N.-P.; software, A.-J.M.-G.; validation, A.-J.M.-G.; formal analysis, G.G.-G.; investigation, A.-J.M.-G., F.-J.H.-L., M.R.N.-P. and G.G.-G.; data curation, G.G.-G.; writing — original draft preparation, A.-J.M.-G., F.-J.H.-L., M.R.N.-P. and G.G.-G.; writing-review and editing, A.-J.M.-G., F.-J.H.-L., M.R.N.-P. and G.G.-G.; visualization, A.-J.M.-G., F.-J.H.-L., M.R.N.-P. and G.G.-G.; supervision, A.-J.M.-G., F.-J.H.-L., M.R.N.-P. and G.G.-G. All authors have read and agreed to the published version of the manuscript.

Funding: Ministry of Education, Culture and Sport of the Government of Spain (Project reference: FPU17/05952).

Acknowledgments: We thank the AREA research group for their involvement in this study.

Conflicts of Interest: The authors declare no conflict of interest.

\section{References}

1. Moreno-Guerrero, A.J.; López-Belmonte, J.; Marín-Marín, J.A.; Soler-Costa, R. Scientific Development of Educational Artificial Intelligence in Web of Science. Future Internet 2020, 12, 124. [CrossRef]

2. Lin, J.; Yu, W.; Zhang, N.; Yang, X.; Zhang, H.; Zhao, W. A survey on internet of things: Architecture, enabling technologies, security and privacy, and applications. IEEE Internet Things J. 2017, 4, 1125-1142. [CrossRef]

3. Zhang, Y.; Ren, J.; Liu, J.; Xu, C.; Guo, H.; Liu, Y. A survey on emerging computing paradigms for big data. Chin. J. Electron. 2017, 26, 1-12. [CrossRef]

4. Bansal, S.; Kumar, D. IoT ecosystem: A survey on devices, gateways, operatingA systems, middleware and communication. Int. J. Wirel. Inf. Netw. 2020, 27, 340-364. [CrossRef] 
5. Yu, W.; Liang, F.; He, X.; Hatcher, W.G.; Lu, C.; Lin, J.; Yang, X. A survey on the edge computing for the Internet of Things. IEEE Access 2017, 6, 6900-6919. [CrossRef]

6. Sun, X.; Ansari, N. EdgeIoT: Mobile edge computing for the Internet of Things. IEEE Commun. Mag. 2016, 54, 22-29. [CrossRef]

7. Sha, K.; Yang, T.A.; Wei, W.; Davari, S. A survey of edge computing-based designs for iot security. Digit. Commun. Netw. 2020, 6, 195-202. [CrossRef]

8. International Telecommunication Union (ITU). Recommendation ITU-T Y.2060. 2012. Available online: http://handle.itu.int/11.1002/1000/11559 (accessed on 5 September 2020).

9. Ashton, K. That 'internet of things' thing. RFiD J. 2009, 22, 97-114.

10. Romero-Rodríguez, J.M.; Alonso-García, S.; Marín-Marín, J.A.; Gómez-García, G. Considerations on the Implications of the Internet of Things in Spanish Universities: The Usefulness Perceived by Professors. Future Internet 2020, 12, 123. [CrossRef]

11. Chaouchi, H.; Bourgeau, T. Internet of Things: Building the New Digital Society. IoT 2020, 1, 1-4. [CrossRef]

12. Gabbai, A. Kevin Ashton Describes "The Internet of Things". 2015. Available online: https://www. smithsonianmag.com/innovation/kevin-ashton-describes-the-internet-of-things-180953749/ (accessed on 19 April 2020).

13. Wang, T.; Zhang, G.; Liu, A.; Bhuiyan, M.Z.A.; Jin, Q. A secure IoT service architecture with an efficient balance dynamics based on cloud and edge computing. IEEE Internet Things J. 2018, 6, 4831-4843. [CrossRef]

14. El-Sayed, H.; Sankar, S.; Prasad, M.; Puthal, D.; Gupta, A.; Mohanty, M.; Lin, C.T. Edge of things: The big picture on the integration of edge, IoT and the cloud in a distributed computing environment. IEEE Access 2017, 6, 1706-1717. [CrossRef]

15. Kono, T.; Taito, Y.; Hidaka, H. Essential Roles, Challenges and Development of Embedded MCU Micro-Systems to Innovate Edge Computing for the IoT/AI Age. IEICE Trans. Electron. 2020, 103, 132-143. [CrossRef]

16. Wu, Q.; Wu, J.; Shen, J.; Yong, B.; Zhou, Q. An edge based multi-agent auto communication method for traffic light control. Sensors 2020, 20, 4291. [CrossRef]

17. Yong, B.; Xu, Z.; Wang, X.; Cheng, L.; Li, X.; Wu, X.; Zhou, Q. IoT-based intelligent fitness system. J. Parallel Distrib. Comput. 2018, 118, 14-21. [CrossRef]

18. Elazhary, H. Internet of Things (IoT), mobile cloud, cloudlet, mobile IoT, IoT cloud, fog, mobile edge, and edge emerging computing paradigms: Disambiguation and research directions. J. Netw. Comput. Appl. 2019, 128, 105-140. [CrossRef]

19. Abbasy, M.B.; Quesada, E.V. Predictable influence of IoT (Internet of Things) in the higher education. Int. J. Inf. Educ. Technol. 2017, 7, 914-920. [CrossRef]

20. Aldowah, H.; Ul Rehman, S.; Ghazal, S.; Naufal-Umar, I. Internet of things in higher education: A study on future learning. J. Phys. Conf. Ser. 2017, 892, 012017. [CrossRef]

21. Mckinsey By 2025 Internet of Things Applications Could Have $\$ 11$ Trillion Impact. 2015. Available online: http://www.mckinsey.com/mgi/overview/in-the-news/by-2025-internet-of-thingsapplications-couldhave-11-trillion-impact (accessed on 13 September 2020).

22. Morabito, R.; Cozzolino, V.; Ding, A.Y.; Beijar, N.; Ott, J. Consolidate IoT edge computing with lightweight virtualization. IEEE Netw. 2018, 32, 102-111. [CrossRef]

23. Ren, J.; Guo, H.; Xu, C.; Zhang, Y. Serving at the edge: A scalable IoT architecture based on transparent computing. IEEE Netw. 2017, 31, 96-105. [CrossRef]

24. Kumar, S.; Singh, J. Internet of Vehicles over Vanets: Smart and Secure Communication using IoT. Scalable Comput. Pract. Exp. 2020, 21, 425-440. [CrossRef]

25. Ge, S.; Cheng, M.; He, X.; Zhou, X. A Two-Stage Service Migration Algorithm in Parked Vehicle Edge Computing for Internet of Things. Sensors 2020, 20, 2786. [CrossRef] [PubMed]

26. Agarwal, Y.; Jain, K.; Karabasoglu, O. Smart vehicle monitoring and assistance using cloud computing in vehicular Ad Hoc networks. Int. J. Transp. Sci. Technol. 2018, 7, 60-73. [CrossRef]

27. Contreras-Castillo, J.; Zeadally, S.; Guerrero-Ibañez, J.A. Internet of vehicles: Architecture, protocols, and security. IEEE Internet Things J. 2017, 5, 3701-3709. [CrossRef]

28. Greco, L.; Percannella, G.; Ritrovato, P.; Tortorella, F.; Vento, M. Trends in IoT based solutions for health care: Moving AI to the Edge. Pattern Recognit. Lett. 2020, 135, 346-353. [CrossRef] 
29. Mrozek, D.; Koczur, A.; Małysiak-Mrozek, B. Fall Detection in Older Adults with Mobile IoT Devices and Machine Learning in the Cloud and on the Edge. Inf. Sci. 2020, 537, 132-147. [CrossRef]

30. Abdellatif, A.A.; Mohamed, A.; Chiasserini, C.F.; Tlili, M.; Erbad, A. Edge computing for smart health: Context-aware approaches, opportunities, and challenges. IEEE Netw. 2019, 33, 196-203. [CrossRef]

31. Akmandor, A.O.; Jha, N.K. Smart health care: An edge-side computing perspective. IEEE Consum. Electron. Mag. 2017, 7, 29-37. [CrossRef]

32. López-Belmonte, J.; Moreno-Guerrero, A.J.; López-Núñez, J.A.; Pozo-Sánchez, S. Analysis of the Productive, Structural, and Dynamic Development of Augmented Reality in Higher Education Research on the Web of Science. Appl. Sci. 2019, 9, 5306. [CrossRef]

33. Moreno-Guerrero, A.J.; Gómez-García, G.; López-Belmonte, J.; Rodríguez-Jiménez, C. Internet Addiction in the Web of Science Database: A Review of the Literature with Scientific Mapping. Int. J. Environ. Res. Public Health 2020, 17, 2753. [CrossRef]

34. Hinojo-Lucena, F.J.; Dúo-Terrón, P.; Ramos, M.; Rodríguez-Jiménez, C.; Moreno-Guerrero, A.J. Scientific Performance and Mapping of the Term STEM in Education on the Web of Science. Sustainability 2020, 12, 2279. [CrossRef]

35. Moreno-Guerrero, A.J. Estudio bibliométrico de la producción científica en Web of Science: Formación Profesional y blended learning. Pixel-Bit. Rev. Medios Educ. 2019, 56, 149-168. [CrossRef]

36. Martínez, M.A.; Cobo, M.J.; Herrera, M.; Herrera, E. Analyzing the scientific evolution of social work using science mapping. Res. Soc. Work Pract. 2015, 25, 257-277. [CrossRef]

37. Moral-Muñoz, J.A.; Herrera-Viedma, E.; Santisteban-Espejo, A.; Cobo, M.J. Software tools for conducting bibliometric analysis in science: An up-to-date review. Prof. Inf. 2020, 29, 1-20. [CrossRef]

38. Hirsch, J.E. An index to quantify an individual's scientific research output. Proc. Natl. Acad. Sci. USA 2005, 102, 16569-16572. [CrossRef]

39. Cobo, M.J.; López, A.G.; Herrera, E.; Herrera, F. Science mapping software tools: Review, analysis, and cooperative study among tools. J. Am. Soc. Inf. Sci. Technol. 2011, 62, 1382-1402. [CrossRef]

40. López-Belmonte, J.; Segura-Robles, A.; Moreno-Guerrero, A.J.; Parra-González, E. Machine Learning and Big Data in the Impact Literature. A Bibliometric Review with Scientific Mapping in Web of Science. Symmetry 2020, 12, 495. [CrossRef]

41. Rodríguez-García, A.M.; López-Belmonte, J.; Agreda-Montoro, M.; Moreno-Guerrero, A.J. Productive, Structural and Dynamic Study of the Concept of Sustainability in the Educational Field. Sustainability 2019, 11, 5613. [CrossRef]

42. López-Robles, J.R.; Otegi-Olaso, J.R.; Porto, I.; Cobo, M.J. 30 years of intelligence models in management and business: A bibliometric review. Int. J. Inf. Manag. 2019, 48, 22-38. [CrossRef]

43. Montero-Díaz, J.; Cobo, M.J.; Gutiérrez-Salcedo, M.; Segado-Boj, F.; Herrera-Viedma, E. Mapeo científico de la Categoría «Comunicación» en WoS (1980-2013). Comunicar 2018, 26, 81-91. [CrossRef]

44. López-Belmonte, J.; Marín-Marín, J.A.; Soler-Costa, R.; Moreno-Guerrero, A.J. Arduino advances in web of science. A Scientific mapping of literary production. IEEE Access 2020, 8, 128674-128682. [CrossRef]

Publisher's Note: MDPI stays neutral with regard to jurisdictional claims in published maps and institutional affiliations.

(C) 2020 by the authors. Licensee MDPI, Basel, Switzerland. This article is an open access article distributed under the terms and conditions of the Creative Commons Attribution (CC BY) license (http://creativecommons.org/licenses/by/4.0/). 